\title{
A Review of Gear Fault Diagnosis of Planetary Gearboxes Using Acoustic Emissions
}

\author{
Félix Leaman ${ }^{1}\left[\right.$ C Cristián Molina Vicuña ${ }^{2} \cdot$ Elisabeth Clausen ${ }^{1}$
}

Received: 30 October 2020 / Accepted: 20 January 2021 / Published online: 9 February 2021

(c) The Author(s) 2021

\begin{abstract}
Despite the progress made in the last decades in the field of machine condition monitoring, there are still cases where the current state of the art is not enough and new technologies and advanced analysis methods are required to prevent unexpected failures. One example is planetary gearboxes (PGs), which are one of the main components of mechanical transmission systems in heavy-duty machines such as off-highway trucks, electric rope shovels, helicopters and wind turbines. Although those machines are usually equipped with vibration and temperature sensors to detect faults in mechanical components, these technologies might not be able to perform well under certain circumstances. Therefore, the applied investigation on new monitoring technologies in the field of machine condition monitoring is a necessary step. Among those, in this review the acoustic emission technology will be addressed as a tool for fault diagnosis of gear faults in PG.
\end{abstract}

Keywords Acoustic emission $\cdot$ Planetary gearbox $\cdot$ Fault diagnosis $\cdot$ Signal processing $\cdot$ Gear faults

\section{Introduction}

One of the main components of rotating machinery are gearboxes. They are used to transmit the rotational movement from one component to another through gears. Additionally, a rotational speed shift can be achieved by using gears of different sizes and number of teeth. When high rotational speed reduction (or increase) ratios are necessary, the planetary gearboxes (PGs) are commonly preferred. The PG has also the characteristic of having concentric input and output shafts. They are used in a wide range of heavy-duty applications, such as off-highway trucks, electric rope shovels, helicopters and wind turbines. In such applications high dynamic loads are usual, which makes the PGs susceptible to early faults within their design lifetime [8,22]. In particular for those heavy-duty applications, the shutdowns produced by unexpected failures can cause important economic losses

Félix Leaman

fleaman@amt.rwth-aachen.de

1 Institute for Advanced Mining Technologies, RWTH Aachen University, Aachen, Germany

2 Laboratorio de Vibraciones Mecánicas, Departamento de Ingeniería Mecánica, Universidad de Concepción, Concepción, Chile that justify the investment on condition monitoring systems to diagnose them early $[9,28]$.

Most machine condition monitoring systems are nowadays based on the measurement of vibrations [17]. However, due to factors such as complex dynamics, structural resonances or slow rotating components, the effect of a fault could not be well represented by the measured vibration signals [16]. In recent years, the AE technology has been gradually growing in the field of condition monitoring. Some investigations have addressed the potential of the $\mathrm{AE}$ over vibration for gearbox fault diagnosis $[5,6,21,25]$. However, in account of the non-stationary and nonlinear behavior of AE signals [14,32], their analysis is a complex task and the tools used in the vibration analysis are not always applicable. Therefore, the analysis of AE signals in PG is still a relative new research field. The main sources of AE in gear transmissions, its influencing variables and the appropriate signal processing methods are to this day not fully addressed topics. Some insights regarding these topics and applications of gear fault diagnosis of PG using AE are presented throughout this short review paper.

\section{Planetary Gearboxes}

PG is the most common type of epicyclic gearbox. They consist of a central sun gear that meshes with multiple planet 
gears around its perimeter as shown in Fig. 1. At the same time, the planet gears mesh with one fixed internal gear called a ring gear. The planet gears are held in their trajectory by an element called the carrier. Due to its geometry and arrangement, one revolution of the carrier causes multiple revolutions of the sun gear. Another main characteristic of the PG is that the load is shared among several meshing points. This allows a more compact but more complex construction in comparison with conventional fixed-shaft gearboxes.

Several factors such as improper lubrication, manufacturing flaws, misalignment or overload can induce different fault modes in gear transmissions [7,13]. Some of them are: wear, fracture, cracking, scoring and plastic flow.

In order to prevent unexpected gear failures and the associated economical loss, gear fault diagnosis has been an intensive research field for several decades. Researchers have studied the measurement of several physical variables in gearboxes such as vibration, temperature and sound to investigate how a gear fault influences these variables. Moreover, the investigations have proposed analysis methods to detect the sometimes underlying effects produced by a fault in the measured signals. The measurement of one physical variable that has been used increasingly for gear fault diagnosis is the measurement of acoustic emissions (AE). In the following sections the measurement and analysis of AE for gear fault diagnosis with focus on PG are addressed.

\section{Acoustic Emissions in Gear Transmissions}

\subsection{Fundamentals}

$\mathrm{AE}$ is the phenomenon of transient elastic wave generation in materials due to rapid releases of strain energy [12]. Most of the $\mathrm{AE}$ waves have frequencies in the range from $20 \mathrm{kHz}$ to 2 $\mathrm{MHz}$, that is, far beyond the human hearing range. Some typical sources of $\mathrm{AE}$ are: crack propagation, friction, impacts, corrosion and cavitation.

The $\mathrm{AE}$ waves can arrive at the sensor as distinguishable single waves or as waves that are so concentrated that they cannot be distinguished from each other. In the first case one speaks of an AE burst, while in the second case one speaks of continuous AE [3]. In the field of condition monitoring events like crack growth and bearing balls passing over a defect produce AE bursts. Other events like cavitation or asperity contacts between rough surfaces produce continuous AE.

\subsection{Measurement Technology}

Most AE sensors consists of a piezoelectric element attached to a wear plate through an conductive adhesive. The coupling of the AE sensor on the surface to be monitored is a key factor for the success of the analysis. With this purpose a coupling

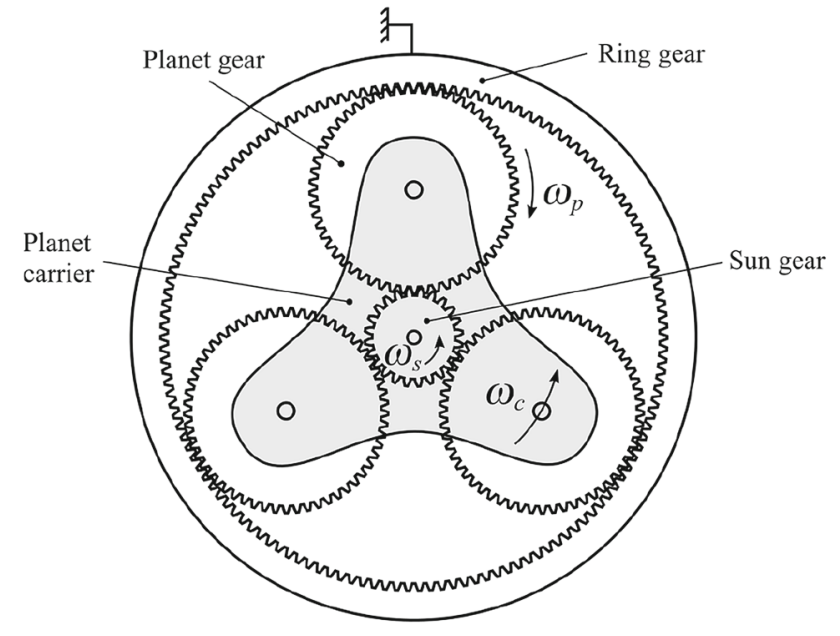

Fig. 1 Components from a planetary gearbox

agent is used to improve the wave transmission from the surface to the sensor. Some used coupling agents are silicone greases, ultrasonic gels and anti-corrosion pastes. The main task of the coupling agent is to avoid the presence of air between the sensor and surface [2].

\subsection{Sources of AE in Gear Transmissions}

In the case of gear transmissions experimental observations have shown that under non-faulty conditions the waveforms of AE signals contain multiple bursts over a continuous AE. Tan and Mba [24,26,27] investigated the sources of AE on parallel-shaft gearboxes that produce such waveforms. They measured the AE with a sensor located directly on the pinion and the signals were transmitted using a slip ring. Three possible sources of $\mathrm{AE}$ were analyzed: tooth resonance, secondary pressure peak in the lubricant and asperity contacts between the meshing surfaces. After having discarded the first two hypotheses, they reached to the conclusion that the main source of $\mathrm{AE}$ in gear transmissions was asperity contacts during sliding and rolling of the meshing teeth. They also suggested that the rolling was responsible for the generation of AE bursts, while the sliding was responsible for the generation of continuous AE. This affirmation was initially based on the observation that the bursts were approximately separated at the gear mesh period. In additional tests without lubrication, they compared the $\mathrm{AE}$ waveforms generated by gears in a good condition and gears with loss of involute profile due to surface damage. The results showed that the bursts were more visible for gears in a good condition than for those with surface damage, which helps to validate their hypothesis.

In the investigation from Novoa and Vicuña [1] additional sources of $\mathrm{AE}$ in gear transmissions were discussed. They mentioned that surface asperities influence the lubricant 


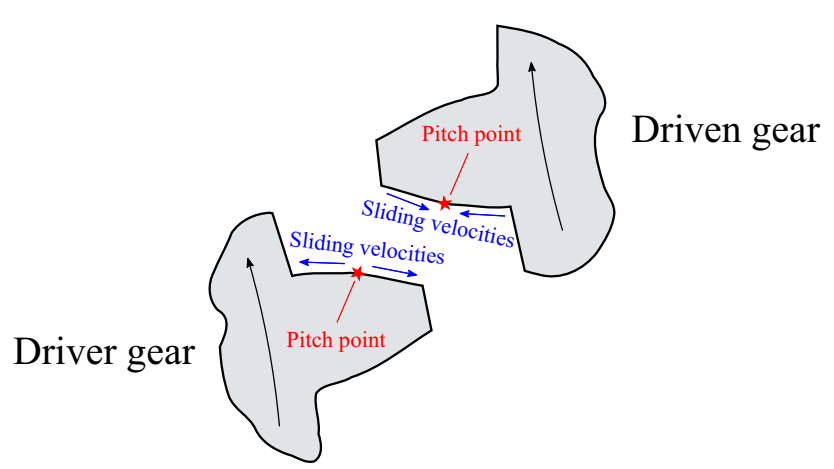

Fig. 2 Sliding and rolling between two meshing teeth

pressure distribution, and thus, extreme pressure perturbations and film breakdown can occur and generate AE bursts. Another hypothesis is the occurrence of a small impact when the teeth mesh, which can cause bursts. Finally, they also proposed that the bursts can originate due to changes in the sliding and rolling conditions of the meshing teeth as shown in Fig. 2.

As described above, a few hypothesis regarding the sources of $\mathrm{AE}$ in gear transmissions that explain the measured waveforms under non-faulty conditions. In spite of the different explanations, it is relatively clear that continuous $\mathrm{AE}$ is caused by the sliding friction between the meshing teeth and that $\mathrm{AE}$ bursts originate as the tooth contact point approaches the pitch point. The presence of gear damage such as wear or cracks may alter or contribute to this phenomenon.

Although most studies for investigating the sources of AE in gear transmissions have been conducted for parallel-shaft gearboxes, the results can also be extrapolated to PG by taking the following considerations. First, in PG there are multiple meshing points of the planet gears with the sun and the ring gear. Therefore, the AE measured by a sensor will be a contribution of all the meshing processes. Second, in PG the distance between meshing points and a stationary sensor on the ring gear changes periodically. Therefore, an amplitude modulation of the measured AE should be expected. This modulation will have a fundamental frequency equal to the carrier rotating frequency times the number of planet gears if all planet gears produce $\mathrm{AE}$ with the same characteristics. However, if the interactions of the planet gears are not equal, the fundamental frequency will be only at the carrier rotating frequency.

\subsection{Influence of Operating Conditions}

Since the values from signal features used for gearbox diagnostics can be altered by the change of operating conditions such as rotational speed, load or temperature, the effects of these conditions must be taken into account for fault diagnosis. Some investigations on this topic are presented below.
In the experiments from Tan and Mba [27] they mentioned the significant influence of the rotational speed on the AE levels during isothermal conditions. As the speed was increased, so did the AE RMS value. Although according to the elastohydrodynamic theory an increase in the speed will cause a thicker lubricant film (and thus less asperity contacts), they attributed the observed behavior to the higher strain rate of asperity contacts. The increase of the AE RMS value was significantly higher when the speed was increased in the lowspeed range than when it was increased in the high-speed range. They argued that at higher speeds the thicker lubricant film effect will be predominant over the higher strain rate of asperity contacts. They even suggested that at some point the AE levels would be in fact reduced. Regarding the influence of load, their results showed that the AE activity is practically unaffected by it.

In other experiments from Tan and Mba [24] AE measurements on a gearbox with a faulty tooth were carried out. After the analysis of the AE waveforms, they concluded that the AE bursts with the largest amplitudes were not always generated as the faulty tooth meshed. They attributed this limitation to the influence of the lubricant temperature on the $\mathrm{AE}$ activity. The temperature changes the lubricant viscosity and thus the lubricant film thickness. Based on the research from Dornfeld and Handy [4], they suggested that the changes in the AE RMS values due to the changes in the lubricant film thickness was a consequence of the asperity contacts as a main source of AE activity in gears. They noticed large variations in the AE RMS values for up to several hours until the lubricant temperature reached the equilibrium. The reason for this was attributed to two contrary effects. On one hand, as the temperature increases the lubricant film thickness decreases allowing more asperity contacts. On the other hand, the meshing surfaces decrease their roughness when they come in contact due to abrasion. Also they showed that when the lubricant temperature was constant, the applied load had negligible effect on the AE RMS value.

Regarding the influence of load, experiments from Hamzah and Mba [10] on spur and helical gears showed a consistent increase in the AE RMS values as the load increased. They attributed these results to the influence of the load on the lubricant film thickness under elastohydrodynamic lubrication. For this condition a higher load produces a thinner lubricant film [23] and thus more asperity contacts and $\mathrm{AE}$ activity. They argued that the influence of the load depends on the lubrication condition. Therefore, they used a more accurate estimation of the lubricant film temperature than Tan and Mba [24,27] to approximate the lubricant film thickness. Their estimation indicated that their experiments were carried out under elastohydrodynamic lubrication condition, while the experiments from Tan and Mba [24,27] were carried out actually under boundary lubrication condition. Under this condition there is no significant change in the level of 
asperity contacts between meshing surfaces under increasing load condition, which explains their results.

Vicuña [30] compared the AE RMS values with the lubricant temperature on a PG test bench with a sensor located on the outside of the ring gear. Similarly to Tan and Mba [24], he found large variations in the AE RMS level with an increasing temperature. While in some tests the AE RMS value increased as the temperature also did, in others tests the AE RMS decreased instead. This result recalls the contrary effects mentioned by Tan and Mba [24] regarding the thinning of the lubricant film and the surfaces smoothing due to abrasion. Vicuña [30] argued that the global behavior of the $\mathrm{AE}$ activity will depend on the contributions from both effects. Regarding the effect of load and rotational speed, he analyzed the magnitudes of gear mesh frequency harmonics of the AE envelope spectrum for different operating conditions. His results indicated that the main factor affecting the AE activity was the rotational speed, while the load had only a small influence at low rotational speeds. At higher rotational speeds the influence of load was negligible. In comparison with the other investigations, his results can be explained in account of a lower specific lubricant film thickness at low rotational speeds.

Novoa and Vicuña [1] also measured the AE on the outside of the ring gear from a PG to investigate the influence of operating conditions. Their results showed that the $\mathrm{AE}$ activity at low rotational speeds is not only influenced by the load, but also by the viscosity of the lubricant. Under the same load and rotational speed conditions, when the PG contained a lubricant of less viscosity (which generates a thinner lubricant film), the AE activity increased. Their results also showed that the rotational speed had a higher influence on increasing the level of AE for the high-speed range than for the low-speed range.

Operating conditions such as rotational speed, load and temperature will affect the $\mathrm{AE}$ activity by means of changes in the lubricant film thickness and in the rate of asperity contacts. Teeth faults such as wear or cracks will modify or be added to the already existing AE. In account of that, an $\mathrm{AE}$ analysis on gearboxes must be able to distinguish between normal $\mathrm{AE}$ and fault-related $\mathrm{AE}$.

\section{Gear Fault Diagnosis}

\subsection{Comparison with Vibration}

The attention to the AE analysis over other traditional techniques for the monitoring of gearboxes is because its ability to detect more incipient damage. A few researchers have carried out comparative experiments regarding the potential of $\mathrm{AE}$ and vibration analysis for gear fault diagnosis. Since most of them have been conducted in fixed-shaft gearboxes rather than in PG, this section describes some studies for both types of gearboxes.

Loutas et al. [21] evaluated several statistical features based on vibration and AE signals to detect crack propagation in an artificially damaged fixed-shaft gearbox under stationary operating conditions. Some features were calculated in the time-domain signal, while others were calculated in the frequency domain. They found out that AE-based features can better follow the damage propagation, especially at early stages. Regionally linear behavior of some AE-based features was observed and gradient changes were associated with changes in the crack propagation rate. The important influence of the lubricant temperature on both vibration and AE-based features was also pointed out.

Vicuña [29] used a cyclostationarity indicator for the analysis of vibration and AE signals measured on a PG test bench to detect a tooth defect of a planet gear. Both sensors (AE and vibration) were installed on the outside of the ring gear. The cyclostationarity analysis was employed to locate the frequency band in which cyclic frequencies of interest were more evident and subsequently an envelope spectra analysis was carried out. The components of interest corresponded to the gear mesh frequency and sidebands. Vicuña concluded that for larger faults the $\mathrm{AE}$ and vibration analysis showed similar evidence regarding the presence of the seeded fault. However, for smaller faults the AE analysis had better results, in the sense that the fault could not be clearly identified by the vibration analysis.

Tan et al. [25] compared the feasibility of using AE RMS value, vibration RMS value and iron concentration to monitor pitting damage in a fixed-shaft gearbox. They concluded that the AE RMS value measured near the pinion had the best performance to represent the evolution of pitting. Moreover, they found a linear correlation between the AE RMS value and the pitting level. However, the correlation was disturbed when the sensor was located on the bearing housing due to the change in the transmission path and the addition of $\mathrm{AE}$ coming from other sources. The performance of iron concentration and vibration RMS value depended upon the load and the pitting level itself.

Eftekharnejad and Mba [5] measured AE and vibration on a helical fixed-shaft gearbox to detect an artificially damaged tooth. In their measurements they installed the AE sensor fixed to the pinion, while the vibration sensor was installed on a bearing pedestal. During the analysis of the waveforms they could clearly identify the defected tooth in account of the higher amplitudes in the AE signals. Moreover, they concluded that the AE RMS values were more sensitive for identification of the seeded fault with different sizes than the vibration levels. 


\subsection{Application Cases}

Most of the application cases of the AE analysis for gear fault diagnosis have been conducted for fixed-shaft gearboxes. However, in account of the important increase in the global wind power generation, every year more researchers focus on the analysis of AE for PG. Some of their investigations are described below.

Novoa and Vicuña [1] used the envelope spectra of AE signals measured on the outside of the ring gear to diagnose defects of sun and planet gears of a PG test bench. They calculated the frequency at which the faulty tooth meshes and corroborated the presence of multiple harmonics of the characteristic fault frequency in the envelope spectra for both cases. For the case of a local fault in the ring gear they did not find any fault symptoms, i.e., they could not establish a difference with the non-faulty case. They also reached to the same conclusions when the tests were repeated for different loads and rotational speeds.

Li et al. [19] used the wavelet transform to process AE signals for localizing a gear fault in a PG by comparing the arrival time of $\mathrm{AE}$ bursts toward the $\mathrm{AE}$ sensors. The appropriate wavelet level to consider was based on a maximum kurtosis criterion and the burst arrival times were considered at the peaks of the wavelet coefficients. Their experimental results confirmed the effectiveness of the presented methodology.

Yoon et al. [33] investigated the use of the empirical mode decomposition (EMD) of AE signals to detect different faults (sun, planet and ring gear) in a PG. Several statistical features were calculated from the intrinsic mode functions (IMF) of the waveforms, envelopes and energy operators of the signals. The measurements were carried out under different operating conditions. Three types of classifiers were trained to distinguish among the faults. The results showed that, depending on the fault, only certain features made the classifiers achieve good performance scores.

He et al. [11] used the Hilbert-Huang transform, which is based on the EMD, to extract fault-related features from $\mathrm{AE}$ and vibration signals. Features such as RMS value, kurtosis and peak amplitude were calculated from the IMF most related to the gear mesh frequency. They proposed to use the calculated features for training of a K-nearest neighbor algorithm for fault detection. The approach was successfully tested with data from a split-torque gearbox with tooth surface damage.

Zhang et al. [34] proposed a methodology for planet gear fault localization using AE signals. They used a wavelet transform to locate the compressive waves that have constant propagation speed at any frequency band. Afterward, they used the wavelet coefficients to estimate the AE burst arrival times toward three AE sensors located on the outside of the ring gear and thus to estimate the fault localization by geometrical analysis. The approach was tested with $\mathrm{AE}$ signals measured from a PG with a chipped tooth.

Leaman [18] analyzed AE measurements from two cases with sensors installed on the outside of the ring gear. The first case was a PG test bench with a worn tooth in the ring gear and the second case was a PG from a wind turbine with a cracked tooth also in the ring gear. For the first case his results indicated that the worn tooth produced an amplitude modulation of the AE signals that can be detected in the envelope spectrum after the use of an appropriate signal processing method. Proposed methods to achieve this task were the wavelet transform or the EMD. For the second case his results indicated that the cracked tooth did not produce noticeable amplitude modulations. However, the AE bursts originating from the cracked tooth had different characteristics of shape and frequency than $\mathrm{AE}$ bursts originating from other healthy teeth.

Khazaee et al. [15] employed fused AE and vibration signal features to train an artificial neural network for classification of gear faults in PG. Together with a healthy case, they considered a ring gear with a cut tooth, a ring gear with a cracked tooth and a planet gear with a worn tooth. Their results indicated that fusion of features considerably improve the performance of the classifier to recognize different types of faults. Accordingly, higher performance scores were achieved with the fused features than for independent features based solely on AE or vibration.

Liu et al. [20] proposed an improvement of the variational mode decomposition method to increase the performance of the method for gear fault diagnosis. In order to test their approach, they analyzed AE and vibration signals from a PG test bench considering two types of faults (crack and pitting) for three different components (sun gear, planet gear and ring gear). In their experiments the sensors were located on the outside of the ring gear. Their results showed that the IMF of the AE had different energies depending on the type and position of the fault, while the energies of the IMF of vibration were very similar for the tested cases. Based on these results, they pointed out the good potential of the approach and provided future research guidelines.

Vicuña and Höweler [31] proposed a method to reduce the amount of $\mathrm{AE}$ data required for gearbox fault diagnosis without losing the most relevant information. The method consisted of creating a simplified version of the AE signal based on the amplitude and occurrence of the AE bursts. They tested the method with data from a PG test bench with a planet gear that had surface damage on a tooth. Their results showed that the spectral analysis of the simplified signal offered similar information as the envelope spectrum. Therefore, frequency components related to planet gear fault were identifiable. 


\section{Discussion and Perspectives}

Several recent investigations have addressed the good potential of the $\mathrm{AE}$ analysis for gearbox condition monitoring, sometimes even highlighting its better potential than other classical techniques. However, there are still vast number of challenges to make the $\mathrm{AE}$ a widely used technique in the field of gearbox condition monitoring. Within this review, the topic of gear fault diagnosis of PG using AE measurements was addressed. Topics such as location of the AE sensor, influence of operating conditions, analysis of AE signals and suggested research guidelines are discussed below.

The measurement location of $\mathrm{AE}$ in gearboxes is a key point for a successful analysis. The AE waves are highly attenuated by interfaces and long trajectories. Therefore, the AE sensor should be located as near as possible to the component under examination. The pioneering investigations in the field of gear fault diagnosis using $\mathrm{AE}$ in parallel-shaft gearboxes were conducted with sensors located directly on the gears. In these investigations the signals were effectively transmitted to the measurement system using a slip ring. A configuration with sensors located on the bearings (a common case for vibration analysis) did not achieve satisfactory results. This could be attributed to the higher number of interfaces that an $\mathrm{AE}$ wave must cross from gear to bearing. A configuration with sensors located on the gearbox case is also not suitable for parallel-shaft gearboxes, since this position is not in direct contact with the gears. However, in PG the outside of the ring gear is a suitable measurement location for AE since it is in direct contact with the planet gears, which in turn are in direct contact with the sun gear. As shown throughout this review, many investigations have been successful by using this location for AE measurement.

Operating conditions can have a high influence on the generated AE in gearboxes. Results from several investigations have suggested that the lubrication regime of the gears plays an important role for the evaluation of the AE signals. Depending on the lubrication regime, operating conditions such as temperature, load and rotational speed can affect the AE signals in different ways. Although some investigations have addressed this influence, the phenomenon is not yet fully understood. For instance, how the presence of a fault modifies the relationship among lubrication regime, operating conditions and $\mathrm{AE}$ is still an open question. A deeper understanding of this influence is key in order to spread the use of the AE technique in condition monitoring systems. In account of this, future research should address the topic of how the detection of a gear fault is affected by the different operating conditions of the gearbox.

The analysis of AE signals benefits particularly from the use of signal processing methods capable of dealing with non-stationary and nonlinear signals. Methods based on the wavelet transform, cyclostationarity and the EMD have been used as tools to improve the analysis of the AE signals from PG.

The potential of the methods based on the wavelet transform transform lies in its own nature. The wavelet coefficients represent the similarity between the original signal and the mother wavelet for different time delays. Since the mother wavelet is of an impulsive nature, the wavelet coefficients will be increased by the presence of AE bursts, even the ones buried in noise. Therefore, a better signal representation in the frequency domain than the one obtained with a Fourier transform can be achieved. Moreover, wavelet transforms, such as the wavelet packet decomposition, can offer a better joint time-frequency resolution than the one obtained with the short-time Fourier transform.

Methods based on the EMD decompose the raw AE signal into IMF that reflect the local time-scale characteristics of the signal. Moreover, these IMF have well-behaved Hilbert transforms, from which better approximations of the envelope can be calculated. For the case of AE analysis, this decomposition also improves the representation of the envelope in the frequency domain based on the Fourier transform. A reason for this is that the IMF are mono-component signals instead of signals of impulsive nature such as AE signals. One downside of the application of methods based on the EMD to AE analysis is the calculation time. Since AE signals are sampled with very high frequency rates, the decomposition of the signal into IMF involves much time. An alternative for obtaining the envelope is the demodulation of a band-passed signal, which is a well-known technique in vibration analysis. However, in the case of AE the frequency band to carry out the demodulation can still contain too much noise that will affect the correct estimation of the envelope. Although the computation time might not be a problem for specific short measurements, it can become an issue for condition monitoring systems that run continuously. Therefore, a suitable research guideline is to develop a fast EMD method suitable for the analysis of AE signals.

To deal with variable rotational speed PG, which could be a common situation in wind turbines or mining machinery, the use of order tracking is a common procedure within the vibration analysis. However, this technique has not yet been employed and evaluated for the analysis of AE signals to the best knowledge of the authors. Due to the considerable higher sampling rate used for $\mathrm{AE}$ in comparison with vibration, a high-precision order tracking that is robust to small deviations in the encoder should also be focus of future research. Moreover, since the generated AE in gear transmissions highly depends on the rotational speed, this high-precision order tracking should not only consider a mechanism to compensate the variations of rotational speed per se, but also to compensate the associated variations of amplitude.

A fourth research guideline consists of the evaluation of a condition monitoring system for PG based on AE for long 
periods of time. The effects produced by gear faults in the AE signals should be analyzed over extended periods of time and be correlated numerically with fault severity. This point is key for the further development of fault prognosis approaches for gear fault prognosis based on AE. Also technical aspects like degradation of the coupling agent over extended periods of time, robustness of the components in industrial environments and the design of a smart storage methodology of relevant $\mathrm{AE}$ data have to be addressed.

Funding Open Access funding enabled and organized by Projekt DEAL.

Open Access This article is licensed under a Creative Commons Attribution 4.0 International License, which permits use, sharing, adaptation, distribution and reproduction in any medium or format, as long as you give appropriate credit to the original author(s) and the source, provide a link to the Creative Commons licence, and indicate if changes were made. The images or other third party material in this article are included in the article's Creative Commons licence, unless indicated otherwise in a credit line to the material. If material is not included in the article's Creative Commons licence and your intended use is not permitted by statutory regulation or exceeds the permitted use, you will need to obtain permission directly from the copyright holder. To view a copy of this licence, visit http://creativecomm ons.org/licenses/by/4.0/.

\section{References}

1. Barrueto Novoa, A., Molina Vicuña, C.: New aspects concerning the generation of acoustic emissions in spur gears, the influence of operating conditions and gear defects in planetary gearboxes. Insight No-Destruct. Test. Cond. Monit. 58(1), 18-27 (2016)

2. Bernet, C.: Ein Beitrag zur Analyse von Schallemissionssignalen. Ph.D. thesis, RWTH Aachen University (2018) (in German)

3. Deutsches Institut fr Normung: DIN EN 1330-9 Zerst-rungsfreie Prfung - Terminologie - Teil 9: Begriffe der Schallemissionsprfung. DIN, Berlin (2009)

4. Dornfeld, D., Handy, C.: Slip detection using acoustic emission signal analysis. In: Proceedings IEEE International Conference on Robotics and Automation. Raleigh, NC, USA (1987)

5. Eftekharnejad, B., Mba, D.: Seeded fault detection on helical gears with acoustic emission. Appl. Acoust. 70(4), 547-555 (2009)

6. Elasha, F., Greaves, M., Mba, D., Fang, D.: A comparative study of the effectiveness of vibration and acoustic emission in diagnosing a defective bearing in a planetary gearbox. Appl. Acoust. 115(1), 181-195 (2017)

7. Fernandes, P., McDuling, C.: Surface contact fatigue failures in gears. Eng. Fail. Anal. 4(2), 99-107 (1997)

8. García Márquez, F., Tobias, A., Pinar Pérez, J., Papaelias, M.: Condition monitoring of wind turbines: techniques and methods. Renew. Energy 46, 169-178 (2012)

9. Hameed, Z., Ahn, S., Cho, Y.: Practical aspects of a condition monitoring system for a wind turbine with emphasis on its design, system architecture, testing and installation. Renew. Energy 35(2010), 879-894 (2010)

10. Hamzah, R.R., Mba, D.: The influence of operating condition on acoustic emission (AE) generation during meshing of helical and spur gear. Tribol. Int. 42(1), 3-14 (2009)

11. He, D., Li, R., Bechhoefer, E.: Split torque type gearbox fault detection using acoustic emission and vibration sensors. In: 2010
International Conference on Networking. Sensing and Control, pp. 62-66. USA, Chicago, IL (2010)

12. Hellier, C.: Handbook of Nondestructive Evaluation. McGrawHill, New York (2003)

13. Hhn, B., Michaelis, K.: Influence of oil temperature on gear failures. Tribol. Int. 37(2), 103-109 (2004)

14. Kamarthi, S., Kumara, S., Cohen, P.: Flank wear estimation in turning through wavelet representation of acoustic emission signals. ASME J. Manuf. Sci. Eng. 122(1), 12-19 (2000)

15. Khazaee, M., Ahmadi, H., Omid, M., Banakar, A., Moosavian, A.: Feature-level fusion based on wavelet transform and artificial neural network for fault diagnosis of planetary gearbox using acoustic and vibration signals. Insight Non-Destruct. Test. Cond. Monit. 55(6), 323-330 (2013)

16. Kim, Y., Tan, A., Kosse, V.: Condition monitoring of low-speed bearings-a review. Aust. J. Mech. Eng. 6(1), 61-68 (2008)

17. Kuseyri, I.: Condition monitoring of wind turbines: challenges and opportunities. In: 3rd International Symposium on Innovative Technologies in Engineering and Sciences. Valencia, Spain (2015)

18. Leaman, F.: Contributions to the diagnosis and prognosis of ring gear faults of planetary gearboxes using acoustic emissions. Ph.D. thesis, RWTH Aachen University (2020)

19. Li, R., Seçkiner, S., He, D., Bechhoefer, E., Menon, P.: Gear fault location detection for split torque gearbox using AE sensors. IEEE Trans. Syst. Man Cybern. Part C Appl. Rev. 42(6), 1308-1317 (2012)

20. Liu, L., Chen, L., Wang, Z., Liu, D.: Early fault detection of planetary gearbox based on acoustic emission and improved variational mode decomposition. IEEE Sens. J. (2020)

21. Loutas, T.H., Sotiriades, G., Kalaitzoglou, I., Kostopoulos, V.: Condition monitoring of a single-stage gearbox with artificially induced gear cracks utilizing on-line vibration and acoustic emission measurements. Appl. Acoust. 70(9), 1148-1159 (2009)

22. Nower, D.: Online vibration monitoring on electric mining shovels. MaintWorld 2, 8-10 (2013)

23. Szeri, A.: Fluid Film Lubrication. Cambridge University Press, Cambridge (1998)

24. Tan, C.: Identification of the acoustic emission source during a comparative study on diagnosis of a spur gearbox. Tribol. Int. 38(5), 469-480 (2005)

25. Tan, C., Irving, P., Mba, D.: A comparative experimental study on the diagnostic and prognostic capabilities of acoustics emission, vibration and spectrometric oil analysis for spur gears. Mech. Syst. Signal Process. 21(2007), 208-233 (2007)

26. Tan, C., Mba, D.: Correlation between acoustic emission activity and asperity contact during meshing of spur gears under partial elastohydrodynamic lubrication. Tribol. Lett. 20(1), 63-67 (2005)

27. Tan, C., Mba, D.: Experimentally established correlation between acoustic emission activity, load, speed, and asperity contact of spur gears under partial elastohydrodynamic lubrication. Proc. Inst. Mech. Eng. Part J J. Eng. Tribol. 219(6), 401-409 (2005)

28. Tian, Z., Jin, T., Wu, B., Ding, F.: Condition based maintenance optimization for wind power generation systems under continuous monitoring. Renew. Energy 36(2011), 1502-1509 (2011)

29. Vicuna, C.: Contributions to the analysis of vibrations and acoustic emissions for the condition monitoring of epicyclic gearboxes. Ph.D. thesis, RWTH Aachen University (2009)

30. Vicuña, C.: Effects of operating conditions on the acoustic emissions (AE) from planetary gearboxes. Appl. Acoust. 77(2014), $150-158$ (2014)

31. Vicuña, C., Höweler, C.: A method for reduction of acoustic emission (AE) data with application in machine failure detection and diagnosis. Mech. Syst. Signal Process. 97(2017), 44-58 (2017)

32. Yang, Z., Yu, Z., Xie, C., Huang, Y.: Application of Hilbert-Huang transform to acoustic emission signal for burn feature extraction in surface grinding process. Measurement 47(2014), 14-21 (2014) 
33. Yoon, J., He, D.: Planetary gearbox fault diagnostic method using acoustic emission sensors. IET Sci. Meas. Technol. 9(8), 936-944 (2015)

34. Zhang, Y., Lu, W., Chu, F.: Planet gear fault localization for wind turbine gearbox using acoustic emission signals. Renew. Energy 109(2017), 449-460 (2017)
Publisher's Note Springer Nature remains neutral with regard to jurisdictional claims in published maps and institutional affiliations. 